\title{
STABILITY OF SOLUTIONS TO COMPLEX MONGE-AMPÈRE EQUATIONS IN BIG COHOMOLOGY CLASSES
}

\author{
Vincent Guedj AND Ahmed Zeriahi
}

\begin{abstract}
We establish various stability results for solutions of complex Monge-Ampère equations in big cohomology classes, generalizing results that were known to hold in the context of Kähler classes.
\end{abstract}

\section{Introduction}

Let $(X, \omega)$ be a compact Kähler manifold of complex dimension $n \in \mathbb{N}^{*}$. Recall that a $(1,1)$-cohomology class is big if it contains a Kähler current, i.e., a positive closed current which dominates a Kähler form.

Fix a non-negative Radon measure $\mu$ whose total mass $\mu(X)$ equals $\operatorname{vol}(\alpha)$, the volume of $\alpha$.

The systematic study of complex Monge-Ampère equations in big cohomology classes has been initiated in [BEGZ10]. It has been shown there that there exists a unique positive closed current $T_{\mu} \in \alpha$ with full Monge-Ampère mass such that

$$
T_{\mu}^{n}=\mu
$$

if and only if $\mu$ is a non pluripolar measure on $X$, i.e., does not charge pluripolar sets.

The purpose of this note is to study the stability properties of the solution $T_{\mu}$ to this complex Monge-Ampère equation, i.e., to study the continuity properties of the mapping

$$
\mu \mapsto T_{\mu}
$$

We can not expect this mapping to be continuous for the weakest topologies, i.e., when the set of non pluripolar measures (resp. the set of positive currents with full Monge-Ampère masses) is endowed with the weak topology of Radon measures (resp. of positive currents), as the Monge-Ampère operator $T \mapsto T^{n}$ is not continuous either for this weak topology (this observation was made, in a local context, by Cegrell and Kolodziej in [CK94]). On the other hand, we have the following:

Proposition A. Let $\mu_{j}, \mu$ be non pluripolar measures with total mass $\mu_{j}(X)=$ $\mu(X)=\operatorname{vol}(\alpha)$. If $\left\|\mu_{j}-\mu\right\| \rightarrow 0$, then

$$
T_{\mu_{j}} \rightarrow T_{\mu} \text { in the weak sense of currents. }
$$

Here $\left\|\mu_{j}-\mu\right\|$ denotes the total variation of the signed measure $\mu_{j}-\mu$.

Received by the editors July 202012

The authors are partially supported by the French ANR project MACK. 
Fix $\theta \in \alpha$ a smooth closed $(1,1)$-form on $X$ representing the big cohomology class $\alpha$. It follows from the $d d^{c}$-lemma that any positive closed current $T \in \alpha$ decomposes as $T=\theta+d d^{c} \varphi$, for some $\theta$-plurisubharmonic function $\varphi$. We let $P S H(X, \theta)$ denote the set of all such functions and observe that there is a unique $\varphi_{\mu} \in P S H(X, \theta)$ such that $\sup _{X} \varphi_{\mu}=0$ and $T_{\mu}=\theta+d d^{c} \varphi_{\mu}$. In the sequel we let

$$
\operatorname{MA}(\varphi):=\left\langle\left(\theta+d d^{c} \varphi\right)^{n}\right\rangle
$$

denote the (non pluripolar) complex Monge-Ampère measure of $\varphi \in P S H(X, \theta)$. The equation $T_{\mu}^{n}=\mu$ is thus equivalent to the Monge-Ampère equation

$$
\operatorname{MA}\left(\varphi_{\mu}\right)=\mu .
$$

Since the weak convergence of currents $T_{\mu}$ is equivalent to the $L^{1}$-convergence of their normalized potentials $\varphi_{\mu}$, Proposition A can be reformulated as

$$
\left(\left\|\mu_{j}-\mu\right\| \rightarrow 0\right) \Longrightarrow\left(\left\|\varphi_{\mu_{j}}-\varphi_{\mu}\right\|_{L^{1}(X)} \rightarrow 0\right) .
$$

It is natural to try and estimate quantitatively how fast this convergence holds. Our second result yields a quantitative stability property "in energy":

Theorem B. There exists $C_{n}>0$ such that if $0 \geq \psi, \varphi_{1}, \varphi_{2} \in \mathcal{E}^{1}(X, \theta)$ are normalized by $\sup _{X} \varphi_{1}=\sup _{X} \varphi_{2}$, then

$$
\int_{X}\left|\varphi_{1}-\varphi_{2}\right| \mathrm{MA}(\psi) \leq C_{n} \cdot B^{2} \cdot I\left(\varphi_{1}, \varphi_{2}\right)^{2^{-n}}
$$

where $B=\max \left\{1,\left|E\left(\varphi_{1}\right)\right|,\left|E\left(\varphi_{2}\right)\right|,|E(\psi)|\right\}$.

We refer the reader to the first section for the definition of the class $\mathcal{E}^{1}(X, \theta)$ of $\theta$-psh functions $\varphi$ which have finite energy $E(\varphi)>-\infty$. We recall here that the symmetric expression

$$
I\left(\varphi_{1}, \varphi_{2}\right):=\int\left(\varphi_{1}-\varphi_{2}\right)\left(M A\left(\varphi_{2}\right)-M A\left(\varphi_{1}\right)\right) \geq 0
$$

is used to define the important notion of "convergence in energy." Theorem B implies in particular a quantitative estimate on how "convergence in energy" implies "convergence in capacity." Related results were previously obtained in [BBGZ09], the latter being a great source of inspiration for this note.

Let us also stress that when the underlying cohomology class is Kähler, a weaker but quite elegant stability result was previously obtained by Blocki in [B103]. We briefly explain in Section 4 how our result can be used to derive more standard stability estimates in this vein.

Our last result yields the strongest property of stability, assuming stronger properties on the corresponding measures.

Theorem C. Assume $\mu=M A\left(\varphi_{\mu}\right)=f_{\mu} \omega^{n}, \nu=M A\left(\varphi_{\nu}\right)=f_{\nu} \omega^{n}$, where the densities $0 \leq f_{\mu}, f_{\nu}$ are in $L^{p}\left(\omega^{n}\right)$ for some $p>1$ and $\varphi_{\mu}, \varphi_{\nu} \in P S H(X, \theta)$ are normalized 
by $\sup _{X} \varphi_{\mu}=\sup _{X} \varphi_{\nu}=0$. Then

$$
\left\|\varphi_{\mu}-\varphi_{\nu}\right\|_{L^{\infty}(X)} \leq M_{\tau}\left\|f_{\mu}-f_{\nu}\right\|_{L^{1}(X)}^{\tau}
$$

where $M_{\tau}>0$ only depends on upper bounds for the $L^{p}$ norms of $f_{\mu}, f_{\nu}$ and

$$
\tau<\frac{1}{2^{n}(n+1)-1} .
$$

The existence of a unique normalized $\theta$-psh function $\varphi_{\mu}$ with minimal singularities such that $\left(\theta+d d^{c} \varphi_{\mu}\right)^{n}=\mu$ when $\mu$ has $L^{p}$-density, $p>1$, has been established in [BEGZ10, Theorem 4.1], generalizing Kolodziej's celebrated result [Kol98].

It is likely that the exponent $\tau$ we obtain here is not sharp. When $\alpha$ is a Kähler class, a better exponent was obtained by Kolodziej in [Kol03] and later on improved by Dinew-Zhang in [DZ10] (see also [Hiep10] for some other generalization).

Notations. In the whole article we fix

- $(X, \omega)$ a compact Kähler manifold equipped with a Kähler form $\omega$,

- $\alpha \in H^{1,1}(X, \mathbb{R})$ a big cohomology class,

- $\theta$ a smooth closed $(1,1)$-form representing $\alpha$.

\section{Preliminary results on big cohomology classes}

We briefly recall here some material developed in full detail in [BEGZ10].

2.1. Quasi-psh functions. Recall that an upper semi-continuous function

$$
\varphi: X \rightarrow[-\infty,+\infty[
$$

is said to be $\theta$-psh iff $\varphi$ is locally the sum of a smooth and a psh function, and $\theta+d d^{c} \varphi \geq 0$ in the sense of currents, where $d^{c}$ is normalized so that

$$
d d^{c}=\frac{i}{\pi} \partial \bar{\partial}
$$

By the $d d^{c}$-lemma any closed positive $(1,1)$-current $T$ cohomologous to $\theta$ can conversely be written as $T=\theta+d d^{c} \varphi$ for some $\theta$-psh function $\varphi$ which is furthermore unique up to an additive constant.

The set of all $\theta$-psh functions $\varphi$ on $X$ will be denoted by $P S H(X, \theta)$ and endowed with the weak topology, which coincides with the $L^{1}(X)$-topology. By Hartogs' lemma $\varphi \mapsto \sup _{X} \varphi$ is continuous in the weak topology. Since the set of closed positive currents in a fixed cohomology class is compact (in the weak topology), it follows that the set of $\varphi \in P S H(X, \theta)$ normalized by $\sup _{X} \varphi=0$ is compact.

We introduce the extremal function $V_{\theta}$ defined by

$$
V_{\theta}(x):=\sup \left\{\varphi(x) \mid \varphi \in P S H(X, \theta), \sup _{X} \varphi \leq 0\right\} .
$$


It is a $\theta$-psh function with minimal singularities in the sense of Demailly, i.e., we have $\varphi \leq V_{\theta}+O(1)$ for any $\theta$-psh function $\varphi$. In fact it is straightforward to see that the following 'tautological maximum principle' holds:

$$
\sup _{X} \varphi=\sup _{X}\left(\varphi-V_{\theta}\right)
$$

for any $\varphi \in P S H(X, \theta)$.

2.2. Ample locus and regularity of envelopes. The cohomology class $\alpha=\{\theta\} \in$ $H^{1,1}(X, \mathbb{R})$ is said to be big iff there exists a closed $(1,1)$-current

$$
T_{+}=\theta+d d^{c} \varphi_{+}
$$

cohomologous to $\theta$ such that $T_{+}$is strictly positive (i.e., $T_{+} \geq \varepsilon_{0} \omega$ for some $\varepsilon_{0}>0$ ). By Demailly's regularization theorem [Dem92] one can then furthermore assume that $T_{+}$has analytic singularities, that is there exists $c>0$ such that locally on $X$ we have

$$
\varphi_{+}=c \log \sum_{j=1}^{N}\left|f_{j}\right|^{2} \bmod C^{\infty},
$$

where $f_{1}, \ldots, f_{N}$ are local holomorphic functions. Such a current $T$ is then smooth on a Zariski open subset $\Omega$, and the ample locus $\operatorname{Amp}(\alpha)$ of $\alpha$ is defined as the largest such Zariski open subset (which exists by the Noetherian property of closed analytic subsets).

Note that any $\theta$-psh function $\varphi$ with minimal singularities is locally bounded on the ample locus $\operatorname{Amp}(\alpha)$ since it has to satisfy $\varphi_{+} \leq \varphi+O(1)$. Note that $\varphi_{+}$does not have minimal singularities unless $\alpha$ is a Kähler class.

In case $\alpha=\{\theta\} \in H^{1,1}(X, \mathbb{R})$ is a Kähler class, plenty of smooth $\theta$-psh functions are available. When $\alpha$ is both big and nef (i.e., $\alpha$ belongs to the closure of the cone of Kähler classes), a good regularity theory is available thanks to [BEGZ10]. However for a general big class the existence of even a single $\theta$-psh function with minimal singularities that is also $C^{\infty}$ on the ample locus $\operatorname{Amp}(\alpha)$ is unknown.

On the other hand we have the following regularity result of Berman-Demailly on the ample locus [BD09]:

Theorem 2.1. The function $V_{\theta}$ has locally bounded Laplacian on $\operatorname{Amp}(\theta)$.

In particular the Monge-Ampère measure $\mathrm{MA}\left(V_{\theta}\right)$ has $L^{\infty}$-density with respect to Lebesgue measure. More specifically we have $\theta \geq 0$ pointwise on $\left\{V_{\theta}=0\right\}$ and

$$
\operatorname{MA}\left(V_{\theta}\right)=\mathbf{1}_{\left\{V_{\theta}=0\right\}} \theta^{n} .
$$

Since $V_{\theta}$ is quasi-psh this result is equivalent to the fact that the current $\theta+d d^{c} V_{\theta}$ has $L_{\text {loc }}^{\infty}$ coefficients on $\operatorname{Amp}(\alpha)$ and shows in particular (by using the Riesz transform and Morrey inequality) that $V_{\theta}$ is in fact $C^{2-\varepsilon}$ on $\operatorname{Amp}(\alpha)$ for each $\varepsilon>0$. 
2.3. Full Monge-Ampère mass. In [BEGZ10] the non-pluripolar product

$$
\left(T_{1}, \ldots, T_{p}\right) \mapsto\left\langle T_{1} \wedge \cdots \wedge T_{p}\right\rangle
$$

of closed positive $(1,1)$-currents is shown to be well-defined as a closed positive $(p, p)$-current putting no mass on pluripolar sets. In particular given $\varphi_{1}, \ldots, \varphi_{n} \in$ $P S H(X, \theta)$ we define their mixed Monge-Ampère measure as

$$
\operatorname{MA}\left(\varphi_{1}, \ldots, \varphi_{n}\right)=\left\langle\left(\theta+d d^{c} \varphi_{1}\right) \wedge \cdots \wedge\left(\theta+d d^{c} \varphi_{n}\right)\right\rangle .
$$

It is a non-pluripolar positive measure whose total mass satisfies

$$
\int_{X} \operatorname{MA}\left(\varphi_{1}, \ldots, \varphi_{n}\right) \leq \operatorname{vol}(\alpha),
$$

where the right-hand side denotes the volume of the cohomology class $\alpha$. If $\varphi_{1}, \ldots, \varphi_{n}$ have minimal singularities then they are locally bounded on $\operatorname{Amp}(\alpha)$, and the product

$$
\left(\theta+d d^{c} \varphi_{1}\right) \wedge \cdots \wedge\left(\theta+d d^{c} \varphi_{n}\right)
$$

is thus well-defined by Bedford-Taylor [BT82]. Its trivial extension to $X$ coincides with $\operatorname{MA}\left(\varphi_{1}, \ldots, \varphi_{n}\right)$, and we have

$$
\int_{X} \operatorname{MA}\left(\varphi_{1}, \ldots, \varphi_{n}\right)=\operatorname{vol}(\alpha) .
$$

In case $\varphi_{1}=\cdots=\varphi_{n}=\varphi$, we simply set

$$
\operatorname{MA}(\varphi)=\operatorname{MA}(\varphi, \ldots, \varphi)
$$

and say that $\varphi$ has full Monge-Ampère mass iff $\int_{X} \operatorname{MA}(\varphi)=\operatorname{vol}(\alpha)$. We let

$$
\mathcal{E}(X, \theta):=\left\{\varphi \in P S H(X, \theta) \mid \int_{X} \operatorname{MA}(\varphi)=\operatorname{vol}(\alpha)\right\}
$$

denote the set of $\theta$-psh functions with full Monge-Ampère mass. Note that $\theta$-psh functions with minimal singularities have full Monge-Ampère mass, but the converse is not true.

A crucial point is that the non-pluripolar Monge-Ampère operator is continuous along monotonic sequences of functions with full Monge-Ampère mass. In fact we have (cf. [BEGZ10] Theorem 2.17):

Proposition 2.2. The operator

$$
\left(\varphi_{1}, \ldots, \varphi_{n}\right) \mapsto \operatorname{MA}\left(\varphi_{1}, \ldots, \varphi_{n}\right)
$$

is continuous along monotonic sequences of functions with full Monge-Ampère mass. If $\int_{X}\left(\varphi-V_{\theta}\right) \mathrm{MA}(\varphi)$ is finite, then

$$
\lim _{j \rightarrow \infty}\left(\varphi_{j}-V_{\theta}\right) \operatorname{MA}\left(\varphi_{j}\right)=\left(\varphi-V_{\theta}\right) \operatorname{MA}(\varphi)
$$

for any monotonic sequence $\varphi_{j} \rightarrow \varphi$. 
2.4. Weighted energies. Let $\psi \in P S H(X, \theta)$ be a $\theta$-psh function with minimal singularities. Its Aubin-Mabuchi energy is

$$
E(\psi):=\frac{1}{n+1} \sum_{j=0}^{n} \int_{X}\left(\psi-V_{\theta}\right)\left\langle\left(\theta+d d^{c} \psi\right)^{j} \wedge\left(\theta+d d^{c} V_{\theta}\right)^{n-j}\right\rangle .
$$

One can check [BEGZ10] that its Gâteaux derivatives are given by

$$
E^{\prime}(\psi) \cdot v=\int_{X} v \operatorname{MA}(\psi)
$$

showing in particular that the functional $\psi \longmapsto E(\psi)$ is non-decreasing.

Definition 2.3. We let $\mathcal{E}^{1}(X, \theta)$ denote the class of all $\theta$-plurisubharmonic functions $\varphi$ such that

$$
E(\varphi):=\inf _{\psi \geq \varphi} E(\psi)>-\infty
$$

where the infimum is taken over all $\theta$-psh functions $\psi$ with minimal singularities.

Alternatively a function $\varphi$ belongs to $\mathcal{E}^{1}(X, \theta)$ if and only if it belongs to $\mathcal{E}(X, \theta)$ and $\varphi \in L^{1}(X, M A(\varphi))$.

More generally, given $\chi: \mathbb{R} \rightarrow \mathbb{R}$ a convex increasing function such that $\chi(-\infty)=$ $-\infty$, one considers, for $\psi$ with minimal singularities,

$$
E_{\chi}(\psi):=\frac{1}{n+1} \sum_{j=0}^{n} \int_{X} \chi\left(\psi-V_{\theta}\right)\left\langle\left(\theta+d d^{c} \psi\right)^{j} \wedge\left(\theta+d d^{c} V_{\theta}\right)^{n-j}\right\rangle .
$$

This weighted energy is again non-decreasing [BEGZ10, Proposition 2.8], hence the following:

Definition 2.4. We let $\mathcal{E}_{\chi}(X, \theta)$ denote the class of all $\theta$-plurisubharmonic functions $\varphi$ such that

$$
E_{\chi}(\varphi):=\inf _{\psi \geq \varphi} E_{\chi}(\psi)>-\infty
$$

where the infimum is taken over all functions $\psi$ with minimal singularities.

One can easily check that these classes exhaust the class of functions with full Monge-Ampère mass [BEGZ10, Proposition 2.11],

$$
\mathcal{E}(X, \theta)=\bigcup_{\chi} \mathcal{E}_{\chi}(X, \theta)
$$

We finally introduce the symmetric expression

$$
I(\varphi, \psi):=\int_{X}(\varphi-\psi)(\operatorname{MA}(\psi)-\operatorname{MA}(\varphi)) \geq 0,
$$

where the non-negativity can be deduced from the following formula

$$
I(\varphi, \psi)=\sum_{j=0}^{n-1} \int_{\Omega} d(\varphi-\psi) \wedge d^{c}(\varphi-\psi) \wedge\left\langle\left(\theta+d d^{c} \varphi\right)^{j} \wedge\left(\theta+d d^{c} \psi\right)^{n-1-j}\right\rangle .
$$

Definition 2.5. A sequence of functions $\varphi_{j} \in \mathcal{E}^{1}(X, \theta)$ converges in energy towards $\varphi \in \mathcal{E}^{1}(X, \theta)$ if $I\left(\varphi_{j}, \varphi\right) \rightarrow 0$ as $j \rightarrow \infty$.

This notion is introduced in [BBGZ09] where it is shown that convergence in energy implies continuity of the complex Monge-Ampère operator. 
2.5. Monge-Ampère capacity. As in [GZ05, BEGZ10] define the Monge-Ampère (pre)capacity as the upper envelope of all measures $\operatorname{MA}(\varphi)$ with $\varphi \in \operatorname{PSH}(X, \theta)$, $V_{\theta}-1 \leq \varphi \leq V_{\theta}$, i.e.,

$$
\operatorname{Cap}(B):=\sup \left\{\int_{B} \operatorname{MA}(\varphi), \varphi \in P S H(X, \theta), V_{\theta}-1 \leq \varphi \leq V_{\theta} \text { on } X\right\} .
$$

for every Borel subset $B$ of $X$.

By definition, a positive measure $\mu$ is absolutely continuous with respect the capacity Cap iff for a Borel set $B \subset X$, $\operatorname{Cap}(B)=0$ implies $\mu(B)=0$. This means exactly that $\mu$ is non-pluripolar in the sense that $\mu$ puts no mass on pluripolar sets. Since Cap is $\sigma$-subadditive, it is in turn equivalent to the existence of a non-decreasing right-continuous function $F: \mathbb{R}_{+} \rightarrow \mathbb{R}_{+}$such that

$$
\mu(B) \leq F(\operatorname{Cap}(B))
$$

for all Borel sets $B$. Roughly speaking the speed at which $F(t) \rightarrow 0$ as $t \rightarrow 0$ measures "how non-pluripolar" $\mu$ is.

Definition 2.6. Fix $\beta>0$. We say that $\mu$ satisfies the condition $\mathcal{H}(\beta)$ if there exists $C_{\beta}>0$ such that for all Borel sets $B \subset X$,

$$
\mu(B) \leq C_{\beta} \operatorname{Cap}(B)^{\beta+1} .
$$

If this holds for all $\beta>0$, we say that $\mu$ satisfies the condition $\mathcal{H}(\infty)$.

Such conditions were introduced by Kolodziej in [Kol98] who showed that measures $\mu=\operatorname{MA}(\varphi)$ satisfying the condition $\mathcal{H}(\beta)$ are such that $\varphi$ is continuous if the cohomology class $\alpha$ is Kähler. He further observed that if $\mu=f \omega^{n}$ has density in $L^{p}$ for some $p>1$, then $\mu$ satisfies condition $\mathcal{H}(\infty)$.

These results were later on extended to the case of big cohomology classes in [EGZ09, Zh06, BEGZ10, EGZ11].

Recall that the complex Monge-Ampère operator $\varphi \mapsto \operatorname{MA}(\varphi)$ is discontinuous for the $L^{1}$-topology. One needs to require a stronger notion of convergence of potentials:

Definition 2.7. A sequence $\left(\varphi_{\ell}\right)$ of $\theta$-plurisubharmonic functions converges in capacity towards $\varphi$ if for all $\varepsilon>0$,

$$
\operatorname{Cap}\left(\left\{\left|\varphi_{\ell}-\varphi\right|>\varepsilon\right\}\right) \rightarrow 0 \text { as } \ell \rightarrow+\infty \text {. }
$$

If a sequence $\left(\varphi_{j}\right)$ of $\mathcal{E}^{1}(X, \theta)$ converges to $\varphi \in \mathcal{E}^{1}(X, \theta)$ in capacity, then $\operatorname{MA}\left(\varphi_{j}\right)$ weakly converges towards $\mathrm{MA}(\varphi)[\mathrm{GZO}, \mathrm{DH}]$. This generalizes previous continuity statements, as monotonic convergence implies convergence in capacity.

\section{Weak stability properties}

In this section we establish the weakest stability property, i.e., Proposition A stated in the introduction. 
3.1. Unstability. We start by observing that one can not expect stability in general. Recall [BEGZ10] that if $\mu$ is a non-negative Radon measure which vanishes on pluripolar sets and whose total mass equals $\operatorname{vol}(\alpha)$, then there exists a unique positive closed current $T_{\mu} \in \alpha$ with full Monge-Ampère mass and such that

$$
\left\langle T_{\mu}^{n}\right\rangle=\mu .
$$

The current $T_{\mu}$ decomposes as $T_{\mu}=\theta+d d^{c} \varphi_{\mu}$, where $\varphi_{\mu} \in P S H(X, \theta)$ is uniquely determined, once normalized by $\sup _{X} \varphi_{\mu}=0$.

One can not expect the operator $\mu \mapsto \varphi_{\mu}$ (or equivalently $\mu \mapsto T_{\mu}$ ) to be continuous, as its inverse operator $\varphi \mapsto\left\langle\left(\theta+d d^{c} \varphi\right)^{n}\right\rangle$ is not either. Here is a variation on a classical local example [Ceg83] of such discontinuous behavior:

Example 3.1. The functions

$$
\psi_{j}\left(z_{1}, z_{2}\right):=\frac{1}{2 j} \log \left[\left|z_{1}^{j}+z_{2}^{j}\right|^{2}+1\right]
$$

are smooth and plurisubharmonic in $\mathbb{C}^{2}$. They form a locally bounded sequence which converges in $L_{\text {loc }}^{1}\left(\mathbb{C}^{2}\right)$ towards

$$
\psi\left(z_{1}, z_{2}\right)=\log \max \left[1,\left|z_{1}\right|,\left|z_{2}\right|\right] .
$$

Observe that the Monge-Ampère measures $\left(d d^{c} \psi_{j}\right)^{2}$ vanish identically, while $\left(d d^{c} \psi\right)^{2}$ is the Lebesgue measure on the real torus $\left\{\left|z_{1}\right|=\left|z_{2}\right|=1\right\}$.

One can globalize this example, working on $X=\mathbb{C P}^{2}$ equipped with its FubiniStudy Kähler form $\theta=\omega_{F S}$. Set

$$
\varphi_{j}[z]=\frac{1}{2 j} \log \left[\left|z_{1}^{j}+z_{2}^{j}\right|^{2}+\left|z_{0}\right|^{2 j}\right]-\log \|z\|,
$$

where $[z]=\left[z_{0}: z_{1}: z_{2}\right]$ denotes the homogeneous coordinates in $\mathbb{C P}^{2}$ and $\left(z_{0}=0\right)$ denotes the hyperplane at infinity, $\mathbb{C P}^{2}=\mathbb{C}^{2} \cup\left(z_{0}=0\right)$.

The functions $\varphi_{j}$ are $\theta$-psh and smooth in $\mathbb{C P}^{2} \backslash S_{j}$, where $S_{j}$ denotes the finite set of points at infinity $\left\{z_{0}=0=z_{1}^{j}+z_{2}^{j}\right\}$. The $\varphi_{j}$ 's converge in $L^{1}\left(\mathbb{C P}^{2}\right)$ towards

$$
\varphi\left(z_{1}, z_{2}\right)=\log \max \left[\left|z_{0}\right|,\left|z_{1}\right|,\left|z_{2}\right|\right]-\log \|z\|,
$$

whose Monge-Ampère measure is again the Lebesgue measure on the torus.

This example is not so satisfactory since the Monge-Ampère measures $\operatorname{MA}\left(\varphi_{j}\right)$ are all supported on the (pluripolar) hyperplane at infinity. We thus propose a slightly more elaborate construction where the approximants are uniformly bounded:

Example 3.2. Using the same notations as in previous example, we set

$$
\Phi_{j}:=\log \left[e^{\varphi_{j}}+e^{-K}\right],
$$

where $K>0$ is a large constant. The reader will easily check that

$$
\theta+d d^{c} \Phi_{j}=\frac{e^{\varphi_{j}} \theta_{\varphi_{j}}+e^{-K} \theta}{e^{\varphi_{j}}+e^{-K}}+\frac{e^{\varphi_{j}-K} d \varphi_{j} \wedge d^{c} \varphi_{j}}{\left[e^{\varphi_{j}}+e^{-K}\right]^{2}} \geq 0,
$$

so that $\Phi_{j}$ are uniformly bounded $\theta$-psh functions on $\mathbb{C P}^{2}$. We use here the shortcuts $\theta=\omega_{F S}$ and $\theta_{u}:=\theta+d d^{c} u$. 
A similar computation can be made for $\Phi:=\log \left[e^{\varphi}+e^{-K}\right]$, showing in particular that

$$
M A(\Phi) \geq \frac{e^{-2 \sqrt{3}}}{\left[e^{-\sqrt{3}}+e^{-K}\right]^{2}} \sigma_{\mathcal{T}}
$$

dominates a multiple of the (normalized) Lebesgue measure $\sigma_{\mathcal{T}}$ on the real torus $\mathcal{T}=\left\{\left|z_{0}\right|=\left|z_{1}\right|=\left|z_{2}\right|\right\}$.

This multiple can be made arbitrarily close to 1 by choosing $K$ large enough. On the other hand, $\operatorname{MA}\left(\Phi_{j}\right)$ can be computed explicitly by using that $\left(d d^{c} \psi_{j}\right)^{2}, d d^{c} \psi_{j} \wedge$ $d \psi_{j}, d d^{c} \psi_{j} \wedge d^{c} \psi_{j}$ are all zero in $\mathbb{C}^{2}$. One can this way verify that any cluster point of $\operatorname{MA}\left(\Phi_{j}\right)$ is different from $\operatorname{MA}(\Phi)$, although $\Phi_{j}$ converges toward $\Phi$.

3.2. Proof of Proposition A. We now prove a qualitative property of stability under a weak domination assumption. Let $\mu_{j}, \mu$ be non negative Radon measures on $X$ which do not charge pluripolar sets and whose total mass equals $\operatorname{vol}(\alpha)$.

Proposition A'. If the measures $\mu_{j}=f_{j} \nu$ are all absolutely continuous with respect to a fixed non pluripolar measure $\nu$ and $f_{j} \rightarrow f$ in $L^{1}(\nu)$, then

$$
T_{\mu_{j}} \rightarrow T_{\mu} \text { in the weak sense of currents, }
$$

where $\mu=f \nu$.

This result can be seen as a generalization of a local result of Cegrell-Kolodziej [CK06] who asked for $f_{j}$ to be uniformly bounded.

Proof. We let $\varphi_{j}, \varphi$ denote the normalized Monge-Ampère potentials,

$$
\mu_{j}=\left(\theta+d d^{c} \varphi_{j}\right)^{n}, \mu=\left(\theta+d d^{c} \varphi\right)^{n}, \text { with } \sup _{X} \varphi_{j}=\sup _{X} \varphi=0 .
$$

We assume that $\mu_{j}=f_{j} \nu, \mu=f \nu$, where $\nu$ vanishes on pluripolar sets and $f_{j} \rightarrow f$ in $L^{1}(\nu)$, and we are going to show that in this case $\left(\varphi_{j}\right)$ converges in $L^{1}(X)$ towards $\varphi$.

By weak compactness, we can assume-up to extracting-that $\varphi_{j} \rightarrow \psi \in \operatorname{PSH}(X, \theta)$, with $\sup _{X} \psi=0$. Extracting again, we can also assume that there exists $g \in L^{1}(\nu)$ such that

$$
f_{j} \leq g \text { for all } j \in \mathbb{N} .
$$

Since the measure $g \nu$ does not charge pluripolar sets, it follows from [BEGZ10, Proposition 3.2] that there exist $\chi: \mathbb{R} \rightarrow \mathbb{R}$ a convex increasing weight and $C>0$ such that $\chi(-\infty)=-\infty$ and for all $j \in \mathbb{N}$,

$$
\int(-\chi)\left(\varphi_{j}-V_{\theta}\right) g d \nu \leq C
$$

This shows that

$$
\int(-\chi)\left(\varphi_{j}-V_{\theta}\right) \mathrm{MA}\left(\varphi_{j}\right) \leq C
$$

hence [BEGZ10, Proposition 2.10] insures that $\psi \in \mathcal{E}_{\chi}(X, \theta)$.

The functions $\psi_{j}:=\left(\sup _{l \geq j} \varphi_{l}\right)^{*} \in \operatorname{PSH}(X, \theta)$ decrease to $\psi$ and satisfy

$$
\operatorname{MA}\left(\psi_{j}\right) \geq\left(\inf _{l \geq j} f_{l}\right) \nu
$$

We infer $\operatorname{MA}(\psi) \geq \mu=f \nu$, when equality since these measures have the same mass $\operatorname{vol}(\alpha)$. 
This shows that $\operatorname{MA}(\psi)=\operatorname{MA}(\varphi)$, hence these normalized potentials have to be equal, by the uniqueness in [BEGZ10, Theorem 3.1].

We finally observe that Proposition A and Proposition A' are equivalent. Indeed if $\mu_{j}=f_{j} \nu$ and $\mu=f \nu$, then by definition

$$
\left\|\mu_{j}-\mu\right\|=\left\|f_{j}-f\right\|_{L^{1}(\nu)},
$$

so that Proposition A' is a particular case of Proposition A.

Conversely, if $\mu_{j}, \mu$ are non pluripolar measures of the same mass $\operatorname{vol}(\alpha)$ such that $\left\|\mu_{j}-\mu\right\| \rightarrow 0$, then

$$
\nu:=\mu+\sum_{j \geq 0} 2^{-j} \mu_{j}
$$

is a well defined non pluripolar Radon measure with respect to which $\mu_{j}, \mu$ are absolutely continuous, thus the hypotheses of Proposition A' are satisfied.

\section{Stability in energy}

4.1. Case of a Kähler class. Our starting point is the following result that is a refinement of [BBGZ09, Lemma 3.12]:

Lemma 4.1. There exists $\kappa_{n}>0$ such that if $0 \geq \varphi_{1}, \varphi_{2}, \psi_{1}, \psi_{2} \in \mathcal{E}^{1}(X, \theta)$ satisfy $E\left(\varphi_{i}\right) \geq-B, E\left(\psi_{i}\right) \geq-B$, then

$$
\left|\int_{X}\left(\varphi_{1}-\varphi_{2}\right)\left(\operatorname{MA}\left(\psi_{1}\right)-\operatorname{MA}\left(\psi_{2}\right)\right)\right| \leq \kappa_{n} B_{+}^{2} I\left(\varphi_{1}, \varphi_{2}\right)^{2^{-n}} I\left(\psi_{1}, \psi_{2}\right)^{2^{-n}}
$$

and

$$
\int_{X} d\left(\varphi_{1}-\varphi_{2}\right) \wedge d^{c}\left(\varphi_{1}-\varphi_{2}\right) \wedge T_{n-1} \leq \kappa_{n} B_{+}^{2} I\left(\varphi_{1}, \varphi_{2}\right)^{2^{-(n-1)}},
$$

where $B_{+}:=\max (1, B)$ and

$$
T_{n-1}:=\sum_{j=0}^{n-1}\left(\theta+d d^{c} \psi_{1}\right)^{j} \wedge\left(\theta+d d^{c} \psi_{2}\right)^{n-1-j} .
$$

A particular case of the second inequality was obtained when $\alpha$ is a Kähler class (which generalized results obtained by Blocki [Bl03] for bounded functions).

Proof. Observe that the first inequality follows from the second one using Stokes formula and Cauchy-Schwarz inequality. We also note that it suffices to establish (4.2) when $\psi_{1}=\psi_{2}=: \psi$, the general case follows by considering $\psi=\left(\psi_{1}+\psi_{2}\right) / 2$.

Set $u:=\varphi_{1}-\varphi_{2}, v:=\left(\varphi_{1}+\varphi_{2}\right) / 2$ and for each $p=0, \ldots, n-1$,

$$
b_{p}:=\int_{X} d u \wedge d^{c} u \wedge \theta_{v}^{p} \wedge \theta_{\psi}^{n-p-1}
$$

where $\theta_{v}:=\theta+d d^{c} v$. Our goal is to bound $b_{0}$ from above, since

$$
b_{0}=\frac{1}{n} \int d\left(\varphi_{1}-\varphi_{2}\right) \wedge d^{c}\left(\varphi_{1}-\varphi_{2}\right) \wedge T_{n-1},
$$

as $\psi=\psi_{1}=\psi_{2}$. 
Using Stokes theorem we obtain

$$
\begin{aligned}
b_{p}= & \int_{X} d u \wedge d^{c} u \wedge \theta_{v}^{p+1} \wedge \theta_{\psi}^{n-p-2}+\int_{X} d u \wedge d^{c} u \wedge d d^{c}(\psi-v) \wedge \theta_{v}^{p} \wedge \theta_{\psi}^{n-p-2} \\
= & b_{p+1}-\int_{X} d u \wedge d^{c}(\psi-v) \wedge d d^{c} u \wedge \theta_{v}^{p} \wedge \theta_{\psi}^{n-p-2} \\
= & b_{p+1}-\int_{X} d u \wedge d^{c}(\psi-v) \wedge \theta_{\varphi_{1}} \wedge \theta_{v}^{p} \wedge \theta_{\psi}^{n-p-2} \\
& +\int_{X} d u \wedge d^{c}(\psi-v) \wedge \theta_{\varphi_{2}} \wedge \theta_{v}^{p} \wedge \theta_{\psi}^{n-p-2},
\end{aligned}
$$

recall that $d d^{c} u=\theta_{\varphi_{1}}-\theta_{\varphi_{2}}$.

Observe that $\theta_{\varphi_{i}} \leq 2 \theta_{v}$, hence Cauchy-Schwarz inequality and (2.3) yield

$$
\left|\int_{X} d u \wedge d^{c}(\psi-v) \wedge \theta_{\varphi_{i}} \wedge \theta_{v}^{p} \wedge \theta_{\psi}^{n-p-2}\right| \leq 2 b_{p+1}^{1 / 2} I(\psi, v)^{1 / 2} .
$$

It follows from [BBGZ09, Lemma 2.7] that $I(\psi, v) \leq a_{n} B_{+}$, where $a_{n}>1$ is a uniform constant, thus

$$
b_{p} \leq b_{p+1}+2\left(a_{n} B_{+}\right)^{1 / 2} \sqrt{b_{p+1}}=h\left(b_{p+1}\right),
$$

where $h(t):=t+2\left(a_{n} B_{+}\right)^{1 / 2} \sqrt{t}$, for $t \geq 0$, is monotone increasing in $t$. Thus

$$
b_{0} \leq h^{n-1}\left(b_{n-1}\right) \leq h^{n-1}\left(I\left(\varphi_{1}, \varphi_{2}\right)\right),
$$

since

$$
b_{n-1} \leq \sum_{j=0}^{n-1} \int d u \wedge d^{c} u \wedge \theta_{\varphi_{1}}^{j} \wedge \theta_{\varphi_{2}}^{n-1-j}=I\left(\varphi_{1}, \varphi_{2}\right) .
$$

Here $h^{n-1}:=h \circ \cdots \circ h$ denotes the $(n-1)$ th-iterate of the function $h$.

Observe that $h(t) \leq C_{1} \sqrt{t}$ for $0 \leq t \leq 1$, where $C_{1}:=1+2\left(a_{n} B_{+}\right)^{1 / 2}$. We infer that if $0 \leq t \leq C_{1}^{-2^{n}}$ then $h^{n-1}(t) \leq C_{1}^{2} t^{2^{-(n-1)}}$. Therefore

$$
b_{0} \leq C_{1}^{2} I\left(\varphi_{1}, \varphi_{2}\right)^{2^{-(n-1)}} \quad \text { if } I\left(\varphi_{1}, \varphi_{2}\right) \leq C_{1}^{-2^{n}} .
$$

When $I\left(\varphi_{1}, \varphi_{2}\right)$ is relatively large, i.e., when $I\left(\varphi_{1}, \varphi_{2}\right)>C_{1}^{-2^{n}}$, we use [BBGZ09, Lemma 2.7] again to bound from above $b_{0} \leq a_{n} B_{+}$, thus obtaining

$$
b_{0} \leq a_{n} B_{+} C_{1}^{2} I\left(\varphi_{1}, \varphi_{2}\right)^{2^{-(n-1)}} .
$$

In both cases we can bound from above $b_{0}$ by $\kappa_{n} B_{+}^{2}$.

When the underlying cohomology class $\alpha$ is Kähler, one can use the classical Poincaré inequality to deduce from Lemma 4.1 a quantitative stability inequality. Indeed assume that $\theta=\omega$ is a Kähler form on $X$ and, for simplicity, that $\operatorname{MA}\left(\varphi_{i}\right)=f_{i} \omega^{n}$ are absolutely continuous with respect to Lebesgue measure, with $L^{2}$-densities.

We can apply the inequality (4.2) with $\psi_{1}=\psi_{2}=0$ and obtain a gradient estimate in terms of the energy deviation: for any $\varphi_{1}, \varphi_{2} \in \mathcal{E}^{1}(X, \omega)$ satisfying $E\left(\varphi_{i}\right) \geq-B$,

$$
\int_{X} d\left(\varphi_{1}-\varphi_{2}\right) \wedge d^{c}\left(\varphi_{1}-\varphi_{2}\right) \wedge \omega^{n-1} \leq \kappa_{n} B_{+}^{2} I\left(\varphi_{1}, \varphi_{2}\right)^{1 / 2^{n-1}}
$$


where

$$
I\left(\varphi_{1}, \varphi_{2}\right)=\int\left(\varphi_{1}-\varphi_{2}\right)\left(f_{2}-f_{1}\right) \omega^{n} \leq\left\|\varphi_{1}-\varphi_{2}\right\|_{L^{2}}\left\|f_{1}-f_{2}\right\|_{L^{2}}
$$

if $\operatorname{MA}\left(\varphi_{i}\right)=f_{i} \omega^{n}$ have $L^{2}$-densities.

We normalize the potentials $\varphi_{i}$ so that $\int_{X} \varphi_{1} \omega^{n}=\int_{X} \varphi_{2} \omega^{n}=0$. It follows then from elementary arguments (see [GZ07]) that the $\varphi_{i}$ 's are uniformly bounded from above on $X$ and their energies are uniformly bounded from below, since

$$
\int\left(-\varphi_{i}\right) M A\left(\varphi_{i}\right)=\int\left(-\varphi_{i}\right) f_{i} \omega^{n} \leq\left\|\varphi_{i}\right\|_{L^{2}}\left\|f_{i}\right\|_{L^{2}}
$$

while Poincaré's inequality yields

$$
\left\|\varphi_{1}-\varphi_{2}\right\|_{L^{2}(X)}^{2} \leq \delta_{n} \int_{X} d\left(\varphi_{1}-\varphi_{2}\right) \wedge d^{c}\left(\varphi_{1}-\varphi_{2}\right) \wedge \omega^{n-1},
$$

for some uniform constant $\delta_{n}>0$. We have thus proved the following stability property:

Proposition 4.2. Let $(X, \omega)$ be a compact Kähler manifold. Let $\varphi_{1}, \varphi_{2} \in \mathcal{E}^{1}(X, \omega)$ be solutions of $\left(\omega+d d^{c} \varphi_{i}\right)^{n}=f_{i} \omega^{n}$, where $\int_{X} f_{i} \omega^{n}=\int_{X} \omega^{n}, f_{i} \in L^{2}(X)$ and $\int\left(\varphi_{1}-\right.$ $\left.\varphi_{2}\right) \omega^{n}=0$. Then

$$
\left\|\varphi_{1}-\varphi_{2}\right\|_{L^{2}(X)} \leq C\left\|f_{1}-f_{2}\right\|_{L^{2}(X)}^{1 /\left(2^{n-1}\right)}
$$

where $C>0$ is a uniform constant.

This result can be seen as a quantitative version of Proposition A' when $\nu=\omega^{n}$. Its purpose is to illustrate, in a simple setting, how Lemma 4.1 can be used to obtain quantitative stability properties. As we shall see in the sequel, similar inequalities will continue to hold in more general contexts.

4.2. The general case. We now go back to our original situation, when the cohomology class $\{\theta\} \in H^{1,1}(X, \mathbb{R})$ is merely big. We start by establishing an important particular case of Theorem B:

Proposition 4.3. There exists $C>0$ such that for every $0 \geq \varphi, \psi \in \mathcal{E}^{1}(X, \theta)$ normalized by $\sup _{X} \varphi=\sup _{X} \psi$,

$$
\|\varphi-\psi\|_{L^{1}(X)} \leq C \cdot B^{2} \cdot I(\varphi, \psi)^{1 / 2^{n}}
$$

where $B:=\max \{1,|E(\varphi)|,|E(\psi)|\}$.

Proof. We can assume without loss of generality that $\nu=\omega^{n}$ is normalized so that $\nu(X):=\int_{X} \omega^{n}=\operatorname{vol}(\alpha)$. If $\varphi \equiv \psi$ there is nothing to prove, so we assume in the sequel that $\varphi \neq \psi$. Reversing the roles of $\varphi, \psi$, we can assume that $\nu(\varphi<\psi)>0$.

Set $Q_{t}:=\{x \in X \mid \varphi(x)>\psi(x)-t\}$. We can find arbitrarily small $t>0$ such that $\nu\left(Q_{t}\right)<\operatorname{vol}(\alpha)$, otherwise $\varphi \geq \psi$ on $X$. Observe also that $\nu\left(Q_{t}\right)>0$ for all $t>0$, otherwise $\varphi \leq \psi-t$ contradicting our normalizing assumption, thus for arbitrarily small $t>0$,

$$
0<a:=\frac{\nu\left(Q_{t}\right)}{\operatorname{vol}(\alpha)}<1
$$


We also set $\left.b:=1-a=\nu\left(X \backslash Q_{t}\right) / \operatorname{vol}(\alpha) \in\right] 0,1[$ and decompose

$$
\|\varphi-\psi\|_{L^{1}(\nu)}=\int_{Q_{t}}(\varphi-\psi) d \nu+\int_{X \backslash Q_{t}}(\psi-\varphi) d \nu+O(t) .
$$

We are going to bound from above each of these integrals by establishing estimates that are independent of $t$ and then let $t$ decrease to zero.

It follows from [BEGZ10] that there exists uniquely determined functions $u, v \in$ $P S H(X, \theta)$ with minimal singularities such that

$$
\operatorname{MA}(u)=a^{-1} \mathbf{1}_{Q_{t}} \nu, \quad \operatorname{MA}(v)=b^{-1} \mathbf{1}_{X \backslash Q_{t}} \nu \quad \text { and } \quad \sup _{X} u=\sup _{X} v=0 .
$$

We also set

$$
U:=a^{1 / n} u+\left(1-a^{1 / n}\right) V_{\theta} \quad \text { and } \quad V:=b^{1 / n} v+\left(1-b^{1 / n}\right) V_{\theta} .
$$

Observe that $U, V \in P S H(X, \theta)$ again have minimal singularities and are still normalized by $\sup _{X} U=\sup _{X} V=0$ (by the tautological maximum principle). Moreover

$$
\operatorname{MA}(U) \geq a \operatorname{MA}(u) \quad \text { while } \operatorname{MA}(V) \geq b \mathrm{MA}(v),
$$

therefore

$$
a \int(\varphi-\psi) \mathrm{MA}(u)+b \int(\psi-\varphi) \mathrm{MA}(v) \leq \int(\varphi-\psi)(\operatorname{MA}(U)-\operatorname{MA}(V)) .
$$

It follows from Lemma 4.1 that the latter is bounded from above by

$$
\kappa_{n} B^{2} I(\varphi, \psi)^{2^{-n}} I(U, V)^{2^{-n}}
$$

where $B=\max (1,-E(\varphi),-E(\psi),-E(U),-E(V))$.

Since $I(U, V)$ is controlled from above if we can bound from below the energies of $U$ and $V$ (see [BBGZ09, Lemma 2.7]), it remains to estimate the latter.

This is in principle very easy, as $U$ and $V$ have minimal singularities, however we want to make clear that the corresponding bounds are independent of $t$ (i.e., independent of $a$ and $b$ ). Since $\operatorname{MA}(u)=g \omega^{n}$ has density in $L^{2}$ (even $L^{\infty}$ ), It follows from [BEGZ10, Theorem 4.1] that

$$
\left\|u-V_{\theta}\right\|_{L^{\infty}(X)} \leq c\|g\|_{L^{2}}^{1 / n} \leq c^{\prime} a^{-1 / n}
$$

since $g=a^{-1} \mathbf{1}_{Q_{t}}$. Therefore

$$
\left\|U-V_{\theta}\right\|_{L^{\infty}(X)}=a^{1 / n}\left\|u-V_{\theta}\right\|_{L^{\infty}(X)} \leq c^{\prime \prime} .
$$

We similarly get a uniform bound from above on $\left\|V-V_{\theta}\right\|_{L^{\infty}(X)}$. Therefore

$$
-c^{\prime \prime \prime} \leq E(U), E(V) \leq 0,
$$

hence the proof is complete. 
We observe the following easy consequence of the previous estimates:

Lemma 4.4. There exists $C_{n}>0$ such that for any $0 \geq \varphi, \psi, u \in \mathcal{E}^{1}(X, \theta)$ normalized by $\sup _{X} \varphi=\sup _{X} \psi$,

$$
\int_{X}(\varphi-\psi) \operatorname{MA}(u) \leq C_{n} \cdot B^{2} \cdot I(\varphi, \psi)^{1 / 2^{n}}
$$

where $B:=\max \{1,|E(\varphi)|,|E(\psi)|,|E(u)|\}$.

Proof. We decompose

$$
\int_{X}(\varphi-\psi) \operatorname{MA}(u)=\int_{X}(\varphi-\psi)\left(\operatorname{MA}(u)-\operatorname{MA}\left(V_{\theta}\right)\right)+\int_{X}(\varphi-\psi) \operatorname{MA}\left(V_{\theta}\right)
$$

and observe that Lemma 4.1 allows to bound from above the first term while the second one is controlled by Proposition 4.3, since $\mathrm{MA}\left(V_{\theta}\right)$ has a bounded density with respect to $\omega^{n}$ by Theorem 2.1 .

We can now prove Theorem B:

Theorem 4.5. There exists $C_{n}>0$ such that if $0 \geq \psi, \varphi_{1}, \varphi_{2}, \in \mathcal{E}^{1}(X, \theta)$ are normalized by $\sup _{X} \varphi_{1}=\sup _{X} \varphi_{2}$, then

$$
\int_{X}\left|\varphi_{1}-\varphi_{2}\right| \mathrm{MA}(\psi) \leq C_{n} \cdot B^{2} \cdot I\left(\varphi_{1}, \varphi_{2}\right)^{2^{-n}}
$$

where $B=\max \left\{1,\left|E\left(\varphi_{1}\right)\right|,\left|E\left(\varphi_{2}\right)\right|,|E(\psi)|\right\}$.

Proof. Set $\varphi:=\sup \left\{\varphi_{1}, \varphi_{2}\right\}$. Observe that $\sup _{X} \varphi=\sup _{X} \varphi_{1}=\sup _{X} \varphi_{2}$ and $\mid \varphi_{1}-$ $\varphi_{2} \mid=2\left(\varphi-\varphi_{1}\right)-\left(\varphi_{2}-\varphi_{1}\right)$, thus

$$
\int_{X}\left|\varphi_{1}-\varphi_{2}\right| \operatorname{MA}(\psi)=2 \int_{X}\left(\varphi-\varphi_{1}\right) \operatorname{MA}(\psi)-\int_{X}\left(\varphi_{2}-\varphi_{1}\right) \operatorname{MA}(\psi) .
$$

The second term on the right-hand side is bounded from above by the desired quantity thanks to Lemma 4.4.

We estimate the first one by using the same lemma, obtaining

$$
\int_{X}\left(\varphi-\varphi_{1}\right) \mathrm{MA}(\psi) \leq C_{n} \cdot D^{2} \cdot I\left(\varphi, \varphi_{1}\right)^{1 / 2^{n}}
$$

where $D:=\max \left\{1,|E(\varphi)|,\left|E\left(\varphi_{1}\right)\right|,|E(\psi)|\right\}$.

Now $|E(\varphi)| \leq\left|E\left(\varphi_{1}\right)\right|$, since $0 \geq \varphi \geq \varphi_{1}$. It therefore suffices to show that $I\left(\varphi, \varphi_{1}\right) \leq I\left(\varphi_{2}, \varphi_{1}\right)$. Recall that

$$
I\left(\varphi, \varphi_{1}\right)=\int_{X}\left(\varphi-\varphi_{1}\right)\left(\operatorname{MA}\left(\varphi_{1}\right)-\operatorname{MA}(\varphi)\right) .
$$

and observe that $\operatorname{MA}(\varphi)=\operatorname{MA}\left(\varphi_{1}\right)$ on the plurifine open set $\left\{\varphi_{1}>\varphi_{2}\right\}$ (see [BT87, GZ05, BEGZ10]). Thus the measure $\operatorname{MA}\left(\varphi_{1}\right)-\operatorname{MA}(\varphi)$ is carried by the Borel set $\left\{\varphi_{2} \geq \varphi_{1}\right\}$ where $\varphi-\varphi_{1}=\varphi_{2}-\varphi_{1}$. Therefore

$$
I\left(\varphi, \varphi_{1}\right)=\int_{X}\left(\varphi_{2}-\varphi_{1}\right)\left(\operatorname{MA}\left(\varphi_{1}\right)-\operatorname{MA}(\varphi)\right) .
$$

In the same way, we obtain

$$
I\left(\varphi, \varphi_{2}\right)=\int_{X}\left(\varphi_{1}-\varphi_{2}\right)\left(\operatorname{MA}\left(\varphi_{2}\right)-\operatorname{MA}(\varphi)\right) .
$$


Adding the two identities yields

$$
I\left(\varphi, \varphi_{1}\right)+I\left(\varphi, \varphi_{2}\right)=I\left(\varphi_{1}, \varphi_{2}\right),
$$

hence, $I\left(\varphi, \varphi_{1}\right) \leq I\left(\varphi_{1}, \varphi_{2}\right)$.

Remark 4.6. We let the reader verify that Proposition 4.3 is a particular case of Theorem 4.5. The latter has the following interesting consequence: if we let $\psi$ be any $\theta$-psh function such that $V_{\theta}-1 \leq \psi \leq V_{\theta}$, then Chebyshev inequality, together with Theorem 4.5, shows that for all $\varepsilon>0$,

$$
\operatorname{Cap}\left(\left\{\left|\varphi_{1}-\varphi_{2}\right|>\varepsilon\right\}\right) \leq \frac{C_{n}}{\varepsilon} B^{2} I\left(\varphi_{1}, \varphi_{2}\right)^{2^{-n}} .
$$

This yields a quantitative estimate on how "convergence in energy" implies "convergence in capacity."

\section{Strong stability}

Let $\mu=f_{\mu} \omega^{n}$ be a non-negative Radon measure which is absolutely continuous with respect to a fixed volume form $\omega^{n}$, with density in $L^{p}$ for some $p>1$. When $\mu(X)=$ $\operatorname{vol}(\alpha)$, it has been shown in [BEGZ10] that the complex Monge-Ampère equation

$$
\left\langle\left(\theta+d d^{c} \varphi_{\mu}\right)^{n}\right\rangle=\mu=f_{\mu} \omega^{n},
$$

has a unique solution $\varphi_{\mu} \in P S H(X, \theta)$ with minimal singularities such that $\sup _{X} \varphi=$ 0 . This is a generalization to the case of big cohomology classes of a celebrated result of Kolodziej [Kol98] (which itself generalized Yau's celebrated $\mathcal{C}^{0}$ a priori estimate [Yau78]).

In this section we prove Theorem $\mathrm{C}$ from the introduction, establishing a quantitative continuity property of the mapping $f_{\mu} \mapsto \varphi_{\mu}$. Since measures with $L^{p}$ densities, $p>1$, satisfy conditions $\mathcal{H}(\beta)$ for all $\beta>0$, Theorem $\mathrm{C}$ is actually a consequence of the following more general result:

Theorem 5.1. Fix $\beta>0$ and assume $\mu, \nu$ are non-negative Radon measures which satisfy the condition $\mathcal{H}(\beta)$ and are normalized so that

$$
\mu(X)=\nu(X)=\operatorname{vol}(\alpha) .
$$

Let $\varphi_{\mu}, \varphi_{\nu}$ be their normalized Monge-Ampère potentials. Then

$$
\left\|\varphi_{\mu}-\varphi_{\nu}\right\|_{L^{\infty}(X)} \leq M_{\tau}\|\mu-\nu\|^{\tau},
$$

where $\tau=\gamma /\left(2^{n}-\gamma\right)$ with $\gamma:=\beta /[n+\beta(n+1)]$.

When $\alpha$ is a Kähler class, Theorem C is due to Kolodziej [Kol03] who obtained a better exponent $\tau$ (see [DZ10] for a sharp improvement of the exponent).

We need the following refinement of a statement proved in [EGZ09] in the context of big and semi-positive cohomology classes: 
Proposition 5.2. Let $\nu$ be a non negative Radon measure which satisfies the condition $\mathcal{H}(\infty)$. Let $\mu=f \nu$, where $0 \leq f \in L^{p}(X, \nu)$ with $p>1$ and $\mu(X)=\operatorname{vol}(\alpha)$. Fix $\varphi, \psi \in$ $P S H(X, \theta)$ such that $\sup _{X} \varphi=\sup _{X} \psi$ and $\mathrm{MA}(\varphi)=\mu$. Then for any $0<\gamma<\frac{1}{n q+1}$,

$$
\sup _{X}(\psi-\varphi)_{+} \leq M\left\|(\psi-\varphi)_{+}\right\|_{L^{1}(X, \nu)}^{\gamma},
$$

where $M>0$ only depends on $\gamma$ and a bound on the $L^{p}$-norm of $f$.

Here $u_{+}=\max (u, 0)$ denotes as usual the maximum of $u$ and 0 .

Let us stress that this relatively technical statement has interesting applications (see e.g [DDGHKZ11] where it is used to establish Hölder-continuity properties of Monge-Ampère potentials). It is an immediate consequence of the following slightly more general (and more technical) result:

Proposition 5.3. Let $\varphi, \psi$ be $\theta$-plurisubharmonic functions such that

$$
-M_{0}+V_{\theta} \leq \sup \{\varphi, \psi\} \leq V_{\theta},
$$

for some $M_{0}>0$. Assume that $\mu:=\left(\theta+d d^{c} \varphi\right)^{n}$ satisfies the condition $\mathcal{H}(\beta)$ for some $\beta>0$. Then there exists $A_{0}=A_{0}\left(\beta, M_{0}\right)$ such that for any $r>0$ we have

$$
\sup _{X}(\psi-\varphi)_{+} \leq A_{0}\left\|(\psi-\varphi)_{+}\right\|_{L^{r}(\mu)}^{\gamma} \text { with } \gamma=\frac{\beta r}{n+\beta(n+r)} .
$$

Moreover if $\mu=f \nu$, where $\nu$ a Borel measure and $f \in L^{p}(\nu), p>1$, then there exists $0<A_{1}=A_{1}\left(\beta, M_{0}, p\right)$ such that

$$
\sup _{X}(\psi-\varphi)_{+} \leq A_{1}\|f\|_{L^{p}(\nu)}^{\gamma q}\left\|(\psi-\varphi)_{+}\right\|_{L^{1}(\nu)}^{\gamma^{\prime}}, \quad \text { with } \gamma^{\prime}=\frac{\beta}{q n+\beta(n q+1)},
$$

where $1 / p+1 / q=1$ and $(\psi-\varphi)_{+}:=\max (\psi-\varphi, 0)$.

Although the proof is very close to that of Propositions 2.6 and 3.1 in [EGZ09], we briefly sketch it for the convenience of the reader.

Proof. Observe first that $(\psi-\varphi)_{+}=\sup \{\varphi, \psi\}-\varphi$ on $X$. So up to replacing $\psi$ by $\sup \{\varphi, \psi\}$, we can assume that $\psi \geq \varphi$ and $\psi$ satisfies the condition $-M_{0}+V_{\theta} \leq \psi \leq V_{\theta}$ on $X$.

Using the "big" comparison principle from [BEGZ10] and arguing exactly as in Proposition 2.6 in [EGZ09], we conclude that there is a constant $B_{0}>0$ such that for any $\varepsilon \in] 0,1]$

$$
\sup _{X}(\psi-\varphi) \leq \varepsilon+B_{0}\left(\operatorname{Cap}(\{\psi-\varphi>\varepsilon\})^{\beta / n} .\right.
$$

The proof of [EGZ09, Proposition 2.6] (cf equation (3) p.616) shows that

$$
\varepsilon^{n} \operatorname{Cap}(\{\psi-\varphi>\varepsilon\}) \leq\left(1+M_{0}\right)^{n} \int_{\{\psi-\varphi>\varepsilon / 2\}} d \mu .
$$

Chebyshev's inequality then yields

$$
\operatorname{Cap}(\{\psi-\varphi>\varepsilon\}) \leq 2^{r} \varepsilon^{-(n+r)}\left(1+M_{0}\right)^{n} \int_{X}(\psi-\varphi)_{+}^{r} d \mu,
$$


for $r>0$ fixed. Therefore

$$
\sup _{X}(\psi-\varphi) \leq \varepsilon+B_{0} 2^{\beta r / n}\left(1+M_{0}\right)^{\alpha} \varepsilon^{-\beta(n+r) / n}\left(\int_{X}(\psi-\varphi)_{+}{ }^{r} d \mu\right)^{\beta / n} .
$$

Choosing $\varepsilon:=\left(\|\psi-\varphi\|_{L^{r}(\mu)} / N\right)^{\gamma}$, where $N$ is an upper bound on $\psi-\varphi$ and $\gamma$ is as in the statement of the proposition yields the desired inequality.

Now if $\mu=f \nu$, where $f \in L^{p}(\nu)$ with $p>1$, Hölder's inequality yields

$$
\int_{X}(\psi-\varphi)^{r}+\mu \leq\|f\|_{L^{p}(\nu)}\left(\int_{X}(\psi-\varphi)^{r q} d \nu\right)^{1 / q} .
$$

The conclusion follows by taking $r:=1 / q$.

Proof of Theorem 5.1. Since $\varphi=\varphi_{\mu}$ and $\psi=\varphi_{\nu}$ have minimal singularities, $\varphi-\psi$ is bounded hence

$$
\begin{aligned}
I(\varphi, \psi) & =\int_{X}(\varphi-\psi)(\operatorname{MA}(\psi)-\operatorname{MA}(\varphi))=\int_{X}(\varphi-\psi) d(\nu-\mu) \\
& \leq\|\varphi-\psi\|_{L^{\infty}(X)}\|\mu-\nu\| .
\end{aligned}
$$

It follows from Proposition 5.3 that

$$
\|\varphi-\psi\|_{L^{\infty}(X)} \leq C_{\beta}\left[\|\varphi-\psi\|_{L^{1}(X, \mu)}^{\gamma}+\|\varphi-\psi\|_{L^{1}(X, \nu)}^{\gamma}\right] .
$$

with $\gamma:=\beta /[n+\beta(n+1)]$. Now Theorem 4.5 implies

$$
\|\varphi-\psi\|_{L^{\infty}(X)} \leq C_{\beta}^{\prime}\left(\|\varphi-\psi\|_{L^{\infty}}\|\mu-\nu\|\right)^{\gamma / 2^{n}}
$$

thus

where $\tau:=\frac{\gamma}{2^{n}-\gamma}$.

$$
\|\varphi-\psi\|_{L^{\infty}(X)} \leq C_{\beta}^{\prime \prime}\|\mu-\nu\|^{\tau}
$$

\section{References}

[BT82] E. Bedford and B.A. Taylor, A new capacity for plurisubharmonic functions, Acta Math. 149(1-2) (1982), 1-40.

[BT87] E. Bedford and B.A. Taylor, Fine topology, Šilov boundary, and $\left(d d^{c}\right)^{n}$, J. Funct. Anal. 72(2) (1987), 225-251.

[BBGZ09] R. Berman, S. Boucksom, V. Guedj and A. Zeriahi, A variational approach to complex Monge Ampère equations, To appear in Publ. Math. I.H.E.S., arXiv: math/0907.4490.

[BD09] R. Berman and J.-P. Demailly, Regularity of envelopes of quasi-plurisubharmonic functions. Perspectives in analysis, geometry, and topology, 39-66, Progr. Math., 296, Birkhäuser/Springer, New York, 2012.

[Bl03] Z. Blocki, Uniqueness and stability for the complex Monge-Ampère equation on compact Kähler manifolds, Indiana Univ. Math. J. 52 (2003), 1697-1701.

[BEGZ10] S. Boucksom, P. Eyssidieux, V. Guedj and A. Zeriahi, Monge-Ampère equations in arbitrary cohomology classes, Acta Math. 205-2 (2010), 199-262.

[Ceg83] U. Cegrell, Discontinuité de l'opérateur de Monge-Ampère complexe, C. R. Acad. Sci. Paris Sér. I Math. 296(21) (1983), 869-871.

[CK94] U. Cegrell and S. Kolodziej, The Dirichlet problem for the complex Monge-Ampère operator, Michigan Math. J. 41(3) (1994), 563-569.

[CK06] U. Cegrell and S. Kolodziej, The equation of complex Monge-Ampère type and stability of solutions, Math. Ann. 334(4) (2006), 713-729. 
[Dem92] J.-P. Demailly, Regularization of closed positive currents and intersection theory, J. Algebraic Geom. 1(3) (1992), 361-409.

[DDGHKZ11] J.P. Demailly, S. Dinew, V. Guedj, P.H. Hiep, S. Kolodziej and A. Zeriahi, Hölder continuous solutions to complex Monge-Ampère equations, Preprint (2011), To appear in J.E.M.S., arXiv:1112.1388.

[DH] S. Dinew and P.H. Hiep, Convergence in capacity on compact Kähler manifolds, Preprint (2009), arXiv:0904.4140.

[DZ10] S. Dinew and Z. Zhang, On stability and continuity of bounded solutions of degenerate complex Monge-Ampère equations over compact Kähler manifolds, Adv. Math. 225(1) (2010), 367-388.

[EGZ09] P. Eyssidieux, V. Guedj and A. Zeriahi, Singular Kähler-Einstein metrics, J. Amer. Math. Soc. 22 (2009), 607-639.

[EGZ11] P. Eyssidieux, V. Guedj and A. Zeriahi, Viscosity solutions to degenerate complex Monge-Ampère equations, Comm. Pure Appl. Math. 64 (2011), 1059-1094.

[GZ05] V. Guedj and A. Zeriahi, Intrinsic capacities on compact Kähler manifolds, J. Geom. Anal. 15(4) (2005), 607-639.

[GZ07] V. Guedj and A. Zeriahi, The weighted Monge-Ampère energy of quasipsh functions, J. Funct. An. 250 (2007), 442-482.

[Hiep10] P.H. Hiep, Hölder continuity of solutions to the Monge-Ampère equations on compact Kähler manifolds, Ann. Inst. Fourier (Grenoble) 60(5) (2010), 1857-1869.

[Kol98] S. Kolodziej, The complex Monge-Ampère equation, Acta Math. 180(1) (1998), 69-117.

[Kol03] S. Kolodziej, The Monge-Ampère equation on compact Kähler manifolds, Indiana Univ. Math. J. 52(3) (2003), 667-686.

[Yau78] S.T. Yau, On the Ricci curvature of a compact Kähler manifold and the complex Monge-Ampère equation, I. C.P.A.M. 31(3) (1978), 339-411

[Zh06] Z. Zhang, On degenerate Monge-Ampère equations over closed Kähler manifolds, Int. Math. Res. Not. 2006, Art. ID 63640, 18 pp.

Institut Universitaire de France \& Institut de Mathématiques de Toulouse, 31062 Toulouse CEDex 09, France

E-mail address: vincent.guedj@math.univ-toulouse.fr

Institut de Mathématiques de Toulouse, 31062 Toulouse Cedex 09, France

E-mail address: ahmed.zeriahi@math.univ-toulouse.fr 